\title{
Effect of 3d Print Layer Orientation on Tensile Properties of Poly(Lactic Acid) Polymer
}

\author{
P.Sethu Ramalingam, K.Mayandi, N.Rajini, Nadir Ayrilmis, S.Rajesh
}

\begin{abstract}
This work investigated the effect of print layer orientation on tensile properties of poly(lactic)acid (PLA) polymer. The size of PLA filament is used as $1.70 \mathrm{~mm}$ diameter. The tensile specimen is modelled in solidworks software as per ISO-527 1BA standard dimensions for plastic materials and saved the data file as STL (Stereo Lithographic) format. The STL file is given as input to the $3 D$ printing machine. The specimen is prepared in 45 degree positions, 90 degree positions and upright positions. The printed samples is tested in Instron universal testing machine. The maximum tensile stress is obtained in 45 degree positions based printed samples due to better load transfer along the printed layer of materials and minimum tensile stress is observed in upright samples due to poor load transformation along the materials. The print layer orientation of the materials is viewed in optical microscope analysis.
\end{abstract}

Keywords: Poly(lactic acid), filament, tensile properties, print layer orientation.

\section{INTRODUCTION}

$3 \mathrm{D}$ printing is a recent techniques for manufacturing a complex shape of the materials and parts. This $3 \mathrm{~d}$ printing techniques is also known as fusion deposit molding and additive manufacturing process and fused filament fabrication process. In this process used the filament for making the materials. This filament is most important materials in $3 \mathrm{D}$ printing process.

This 3D filament is making from polymers and other printing machines. These are ABS (acrylonitrile butadiene Styrene), PLA (poly(lactic)acid), PVA (Poly Vinyl Alcohol, Polyethylene Tetraphthalate (PTFE), HIPS (High Impact Poly Styrene), Nylon and other composite filaments are

Revised Manuscript Received on December 5, 2019

* Correspondence Author

Mr.P.Sethu Ramalingam, Mechanical Engineering, Rajalakshmi Institute of Technology, Chennai, Tamilnadu, India. Email: sethu1412@gmail.com

Dr.K.Mayandi*, Mechanical Engineering Department, Kalasalingam Academy of Research and Education, Virudhunagar, India. Email: k.mayandi@gmail.com

Dr.N.Rajini, Professor, Mechanical Engineering Department, Kalasalingam Academy of Research and Education, Virudhunagar, India. Email: rajiniklu@gmail.com.

Prof. Nadir Ayrilmis, Department of Wood Mechanics and Technology, Faculty of Forestry, Istanbul University-Cerrahpasa, Bahcekoy, Sariyer, 34473, Istanbul, Turkey Email: nadiray@ istanbul.edu.tr.

Dr.S.Rajesh, Mechanical Engineering Department, Kalasalingam Academy of Research and Education, Virudhunagar, India. Email: s.rajesh@klu.ac.in. materials. There are several type of filament are used in 3D

Wood/PLA, Carbon/PLA, Metal/PLA, etc., [1-5]. Each filament is used depends upon the type of applications area. For example, metal/PLA filament is used for making mechanical parts, Nylon is used for making machine parts, toy machine parts mechanical components like gears and bearings, consumer products. PETT is used for food packaging applications areas, because this material is safe for direct contact of food such as cup, kitchen utensils and soda pop bottles making purpose is used. PLA is one of the bio degradable polymers and these PLA filament is used making medicine oriented surgical inplant parts are manufactured such as inplanted screw and pin and rod. This PLA filament is also used for food packaging applications areas such as candy wrappers, disposable table wares. Nowadays 3D printing process is used for making lightweight materials. For example unmanned aerial vehicle wing structure are fabricated are fabricated using fusion deposit molding (FDM) process [6,7]. Moreover the this process has taken very less fabrications time, low cost, easy to change the materials, low maintenance, supervision free operations and low working temperature $[8,9]$.

Our objective of the work is to investigate the effect of print layer orientation direction on tensile properties of PLA polymer and analysis the tensile stress for various positioned based printed materials. The main reason for choosing PLA as filament has biodegradable and eco-friendly to nature. More over this materials is not releasing the harmful odour during printing of materials.

\section{MATERIALS AND METHODS}

\section{A.Materials and Modelling}

PLA filament is used for making the 3D samples. PLA is purchased from Zaxe Pvt. Ltd, Turkey. Initially the sample is designed in modelling software as per ISO 527 1BA standards dimensions and saved as STL (Stereo Litho graphic) format. After that the STL fie is given as input to the 3D printing machines. The making of 3D printing machine by Zaxe Pvt Ltd. Turkey and model is Zaxi X1.

\section{B. Printing Specifications}

Before printing the materials, the print layer thickness is set as $0.2 \mathrm{~mm}$ and melting temperature is $200^{\circ} \mathrm{C}$. The bed temperature of the machine is set as $80^{\circ} \mathrm{C}$. The fill density of the materials is set as $80 \%$ of flow area of the nozzle and printing speed of the machine is maintained as $40 \mathrm{~mm} / \mathrm{sec}$. 


\section{Effect of 3d Print Layer Orientation on Tensile Properties of Poly(Lactic Acid) Polymer}

The material is printed in various print layer orientations directions. These are 45 degree, 90 degree positions and upright positions is should be showed as figure $1 \mathrm{a}, \mathrm{b}$, and $\mathrm{c}$.

\section{Tensile Test}

Tensile test is conducted as per ISO 527-1BA standard. The gauge length of the specimen is set as $50 \mathrm{~mm}$ and the cross head of the machine speed is $5 \mathrm{~mm}$ per min. The dimensions of the specimen is $75 \mathrm{~mm}$ length, $10 \mathrm{~mm}$ width in grip side area and $5 \mathrm{~mm}$ width in gauge length side area and thickness of the materials is $2 \mathrm{~mm}$.

\section{Optical Microscopic Analysis}

The print layer orientations of sample is viewed in Motic microscope analysis. The specimens is grounded on the flat surfaces of microscope and captured the images of outer surfaces and print layer orientations of the materials. All the images are capture with $5 \mathrm{X}$ magnifications factor.
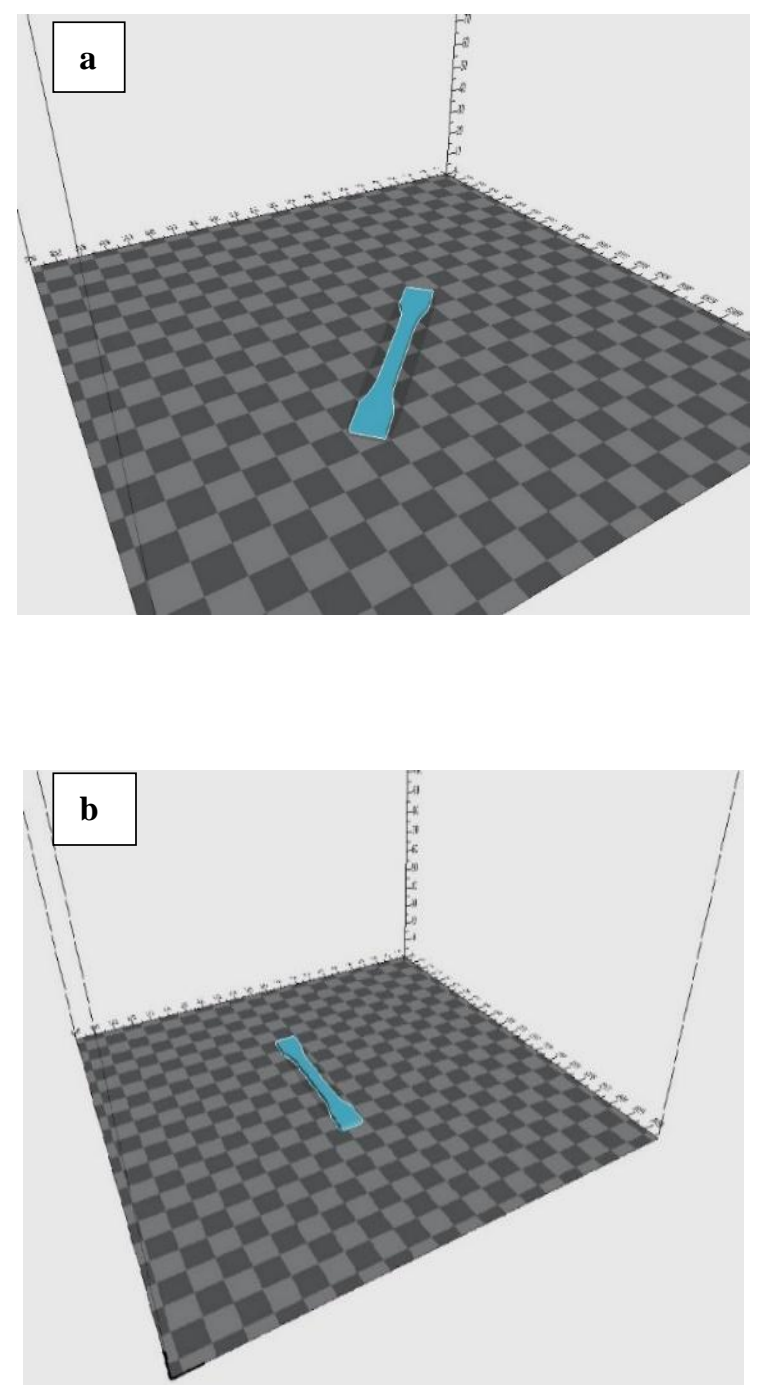

Fig 2 shows the stress strain diagram of 3D printed materials with various print layer orientation. It was shown that the maximum stress is developed on 45 degree position based printed materials and lowest stress is obtained on upright positioned samples. The main reason for variations for the same material is print layer orientation is changed and also loading directions is varied on the printed layer materials. For example in 45 degree positioned materials, the machine is printed one layer is formed in along the axis of the materials and other layer is printed transverse direction of axis of the materials. Similarly the entire layer of the materials is formed alternatively in axis of the materials and transverse direction of the materials it was shown in fig 3a. In 90 degree position based printed materials has the maximum stress as $40.41 \mathrm{MPa}$. In this materials the print layer is formed in +45 degree and -45 degree and it was shown in fig $3 \mathrm{~b}$. The main reason for obtaining lowest value compared to other because the applied loading is transferred in inclined on the printed layers. The tensile properties of various orientation of 3D printed PLA materials is listed on table 1.

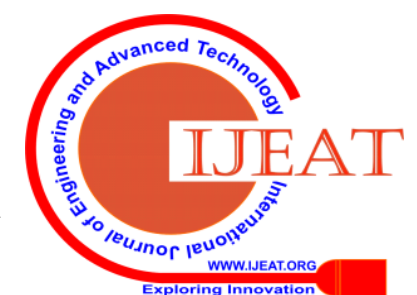


The highest tensile strain is obtained on $90^{\circ}$ printing position based materials and the lowest tensile strain is predicted on 45 degree position based materials. This is happen due to load transfer is varying on print layer of each printed materials.

Table 1. Tensile Properties

\begin{tabular}{|l|l|l|l|l|}
\hline S.L.No & $\begin{array}{l}\text { Printing } \\
\text { Positions }\end{array}$ & $\begin{array}{l}\text { Stress } \\
(\mathrm{MPa})\end{array}$ & $\begin{array}{l}\text { Modulus } \\
(\mathrm{MPa})\end{array}$ & $\begin{array}{l}\text { Strain } \\
(\%)\end{array}$ \\
\hline 1 & $45^{\circ}$ & 45.29 & 1855.5 & 2.53 \\
\hline 2 & $90^{\circ}$ & 40.41 & 612.0 & 3.25 \\
\hline 3 & upright & 25.93 & 1320.0 & 2.80 \\
\hline
\end{tabular}
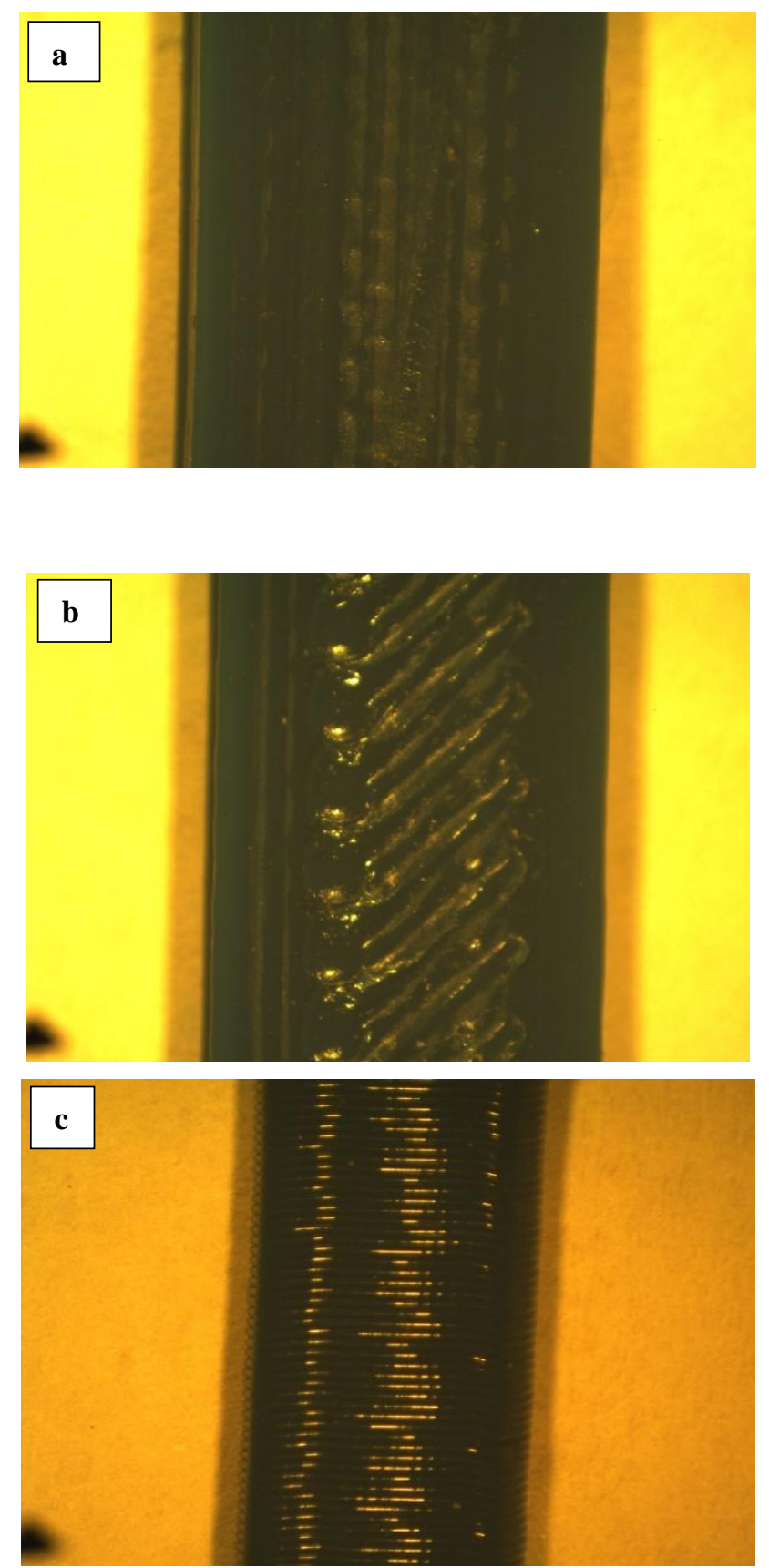

Figure 3. Optical Microscope Images of various print layer orientations. a) Printing from 45 degree positions b) printing from 90 degree positions c) printing from upright positions
Figure 3a-c shows the print layer orientations of various positions based printed materials and the optical microscope images confirmed the printing layer is printed in various orientation on 3D printed materials.Fig 3a shows the printing layer along the axis of materials and transverse direction of the axis. This images (fig3a) obtained from 45 degree positions based materials. When changing the positions of the materials the $3 \mathrm{D}$ printing machine (Zaxe $\mathrm{x} 1$ ) automatically changed its print layer orientations. By changing positions in 90degree the print layer orientations is formed in $+45^{\circ}$ and $-45^{\circ}$ directions is shown in fig $3 \mathrm{~b}$. In upright positions each printing layer is formed in oval shape from bottom to top of the materials. The printing layer of upright positions is shown in fig $3 c$.

\section{CONCLUSION}

The following conclusions were obtained from the above study

- The PLA 3D sample is successfully fabricated as per ISO 527-1BA standard.

- The maximum tensile stress of 3D printed materials is observed in 45 degrees position based materials.

- The maximum tensile stress and tensile modulus is predicted as $45.29 \mathrm{MPa}$ and $1855.5 \mathrm{MPa}$ respectively for 45 degrees printing positions due to better load transfer along the print layer of the materials and minimum tensile stress is observed as $25.93 \mathrm{MPa}$ for upright printing positioned materials. The minimum tensile modulus is observed in 90 degree printing positioned materials due to applying load is acting inclined to the print layer.

- The microscope images confirmed that the print layer is printed in different orientations on the materials.

- When changing the positions of printing, the print layer orientation on the $3 \mathrm{D}$ materials is automatically changed during printing.

\section{REFERENCES}

1. F. Akasheh, et al., "Effect of Build Orientation on the Mechanical Properties and Fracture Behavior of ABS Produced by Fused Deposition Modeling," Microsc. Microanal. 23 (Suppl 1), 2017 doi:10.1017/S1431927617004457

2. I. Gibson, D.W. Rosen, B.Stucker, "Additive manufacturing Technologies", (Springer, NY) 2010.

3. A. Bellini, and S. Güçeri, "Mechanical Characterization of Parts Fabricated using Fused Deposition Modeling," Rapid Prototyping Journal, vol 9(4), 2003, pp. 252-264.

4. Nadir Ayrilmis, et al., "Effect of printing layer thickness on water absorption and mechanical properties of 3D-printed wood/PLA composite materials," The International Journal of Advanced Manufacturing Technology, Vol. 102, June 2019, pp. 2195-2200. 


\section{Effect of 3d Print Layer Orientation on Tensile Properties of Poly(Lactic Acid) Polymer}

5. Patrick Hartunian and Mohsen Eshraghi, "Effect of Build Orientation on the Microstructure and Mechanical Properties of Selective Laser-Melted Ti-6Al-4V Alloy," J. Manuf. Mater. Process. 2018, 2, 69; doi:10.3390/jmmp2040069,

6. S. K. Moon, Y. E. Tan, J. Hwang, and Y. J. Yoon, "Application of 3D printing technology for designing light-weight unmanned aerial vehicle wing structures," Int. J. Precis. Eng. Manuf.- Green Technol., vol. 1, no. 3, 2014, pp. 223-228.

7. Ahn Sung Hoon, Montero Michael, Odell Dan, Roundy Shad, K. Wright Paul, "Anisotropic material properties of fused deposition modelling ABS," Rapid prototyping journal, 8 (4), 248-257, 2002.

8. K. M. Ashtankar, Dr. A. M. Kuthe, Bechu Singh Rathour, "Effect Of Build Orientation On Mechanical Properties Of Rapid Prototyping (Fused Deposition Modelling) Made Acrylonitrile Butadiene Styrene (ABS) Parts" in Proceedings of the ASME 2013 International Mechanical Engineering Congress and Exposition, USA 2013.

9. Rolando Quintana, Jae-Won Choi, Karina Puebla \& Ryan Wicker, "Effects of build orientation on tensile strength for stereolithography-manufactured ASTM D-638 type I specimens" Int $J$ Adv Manuf Technol, vol. 46, 2010, pp. 201-215. DOI 10.1007/s00170-009-2066-z.

\section{AUTHORS PROFILE}

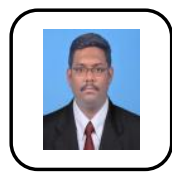

Mr.P.Sethuramalingam is a Ph.D. student at School of Automotive and Mechanical Engineering at Kalasalingam Academy of Research and Education, Krishnankoil, Madurai, India. He is working as an Assistant Professor in Rajalakshmi Institute of Technology (RIT), Chennai. He received his B.E. (2009) Mechanical Engineering from Anna University, Chennai and obtained his M.E. (2013) in Manufacturing from same university. His research interests Additive Manufacturing, polymer composites for medium load applications areas.

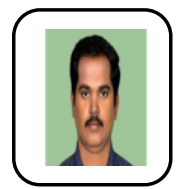

Dr.K.Mayandi received his B.E. (2007) in Mechanical Engineering and M.E. (2011) in Engineering Design from Anna University, Chennai and obtained the Ph.D (2016) from Kalasalingam University, Krishnankoil. He is working as an Associate Professor in the School of Automotive and Mechanical Engineering at Kalasalingam Academy of Research and Education, Krishnankoil, Madurai, India. His research interest on characterization of natural fibres and utilized the natural fibres as reinforcement with polymer composites materials for biodegradable applications. Recently focuses the research on fracture analysis of layered polymer composites materials and also involves the works on fabrication and testing of filament winding polymer composites tube for medium load applications areas. He has published 17 international journals and 2 book chapters published in Elsevier and Wiley publications.

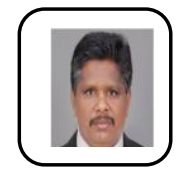

Dr.N.Rajini is working as Professor in the department of Mechanical Engineering in Kalasalingam Academy of Research and Education since 2004. He completed his Ph.D in 2013 from Kalasalingam University. He completed his post graduation in 2000. His area of interest is composite materials. He has international collaborative projects and researches. $\mathrm{He}$ published more than $150 \mathrm{SCI}$ and SCI-Expended articles.

Dr.S.Rajesh is working as an Associate Professor and Head of the Department in the department of Mechanical Engineering at Kalasalingam Academy of Research and Education since 2004. He completed his Ph.D in 2014 from Kalasalingam University. He completed his post-graduation in 2004. His area of interest is machining studies in composite materials.. He published more than $20 \mathrm{SCI}$ and SCI-Expended articles.

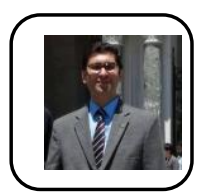

Dr. Nadir Ayrilmis is currently working as Ful Prof.Dr., at Depatment of Wood Mechanics and Technology, Faculty of Forestry, Istanbul University-Cerrahpasa since 1998 and having 21 years of experience in teaching, research in the field of wood technology, wood-based panels and natural-fiber composites, composites, includes raw matarial characteristics of wood and non-wood natural fibers, man-made fibres, production of wood-based composites and natural fiber composites, 3D printed natural fiber/bio polymers, inorganic bonded composites, sandwich-structured wood based composites, fire-retardant treated composites, modification and Treatment of Lignocellulosic Fibres and Solid Wood, cano composites and Nanocellulose fibres. So far he has published 3 book chapters, and more than 165 International journal papers (SCI/SCI-Expanded) in reputed Journals in the field of Wood Sicence and Technology, and Natural Fiber Composites and allied field 